\title{
Prognostic Significance of Preoperative Controlling Nutritional Status Score in Patients Who Underwent Hepatic Resection for Hepatocellular Carcinoma
}

\author{
Bum-Soo Kim \\ Division of Hepatobiliary Surgery and Liver Transplantation, Department of Surgery, Kyung Hee University Hospital, Kyung Hee Univer- \\ sity School of Medicine, Seoul, Korea
}

Received Feb. 23, 2020

Revised May 15, 2020

Accepted May 18, 2020
Malnutrition is common in patients with hepatocellualar carcinoma (HCC), and is associated with postoperative complications after hepatectomy, and also increased mortality. However, there is currently no recommendation for assessment of nutritional status in HCC patients. The controlling nutritional status (CONUT) score has been correlated with prognosis in gastrointestinal cancer patients, but there are few reports on the prognostic significance of the CONUT score in patients who underwent hepatectomy for HCC. Existing results show that patients with high CONUT scores who undergo hepatectomy for HCC have poorer survival outcomes, and experience more complications than other patients. In this paper, we review the literature, and reveal that patients who underwent hepatectomy for HCC with high preoperative CONUT scores had poorer outcomes than those with low CONUT scores. Therefore, we conclude that a preoperative CONUT score may be useful for prognostic prediction in patients with HCC undergoing curative hepatectomy. (J Liver Cancer 2020;20:106-112)

Keywords: Controlling nutritional status score; Hepatocellular carcinoma; Outcomes

\section{INTRODUCTION}

Hepatocellular carcinoma (HCC) is the third-leading cause of cancer-related deaths worldwide. ${ }^{1} \mathrm{HCC}$ is associated with liver cirrhosis in $80-90 \%$ of patients. In addition, these patients typically suffer from malnutrition, which has been associated with a poor prognosis. Consequently, an indicator that could easily and accurately assess malnutrition would be particularly valuable for HCC patients. ${ }^{2-4}$

\section{Corresponding author : Bum-Soo Kim}

Division of Hepatobiliary Surgery and Liver Transplantation, Department of Surgery, Kyung Hee University Hospital, Kyung Hee University School of Medicine, 23 Kyungheedae-ro, Dongdaemun-gu, Seoul 02447, Korea Tel. +82-2-958-8241, Fax. +82-2-966-9366

E-mail; kbs420@hanmail.net

https://orcid.org/0000-0003-2216-0403
Although preoperative diagnoses, surgical techniques, and postoperative management for HCC have improved, clinical outcomes for HCC remain poor, with a 5-year recurrence rate of $70 \%$, even in patients undergoing curative treatment. ${ }^{5}$ Several studies have reported potential prognostic factors for HCC. In contrast to other solid malignancies, the prognosis and treatment options for HCC depend on tumor stage and hepatic functional reserve. ${ }^{6}$ The two most commonly used scoring systems are the Cancer of the Liver Italian Program score, and the Barcelona Clinic Liver Cancer staging classification. ${ }^{7,8}$ However, these scoring systems focus on tumor related factors, liver functional reserve, and physical condition, which are difficult to predict accurately for prognosis.

Recently, both systemic inflammatory responses indicated by the prognostic nutritional index (PNI), and immune-nu-

Copyright $\odot 2020$ by The Korean Liver Cancer Association. All rights reserved.

This is an Open Access article distributed under the terms of the Creative Commons Attribution Non-Commercial License (http://creativecommons.org/licenses/by-nc/4.0/) which permits unrestricted non-commercial use, distribution, and reproduction in any medium, provided the original work is properly cited. 
tritional status determined by the controlling nutritional status (CONUT) score, have been correlated with poor survival in patients with HCC. ${ }^{9-11}$ Herein, we review the literature, and report on the prognostic significance of preoperative CONUT scores in patient clinical outcomes after hepatic resection for HCC.

\section{NUTRITION AND PROGNOSIS FOR HCC}

Recently, several studies have reported cumulative data on systemic inflammatory and nutritional status in cancer progression, and its association with clinical outcomes. ${ }^{12-16}$ Malnutrition is frequent but underdiagnosed in cancer patients, even though one third of cancer patients are malnourished. ${ }^{17}$ Malnutrition is defined as a decline in lean body mass with the potential for functional impairment at multiple levels. ${ }^{18}$ In patients with liver cirrhosis leading to HCC, malnutrition is a common finding and is associated with increased mortality and reduced quality of life. ${ }^{19,20}$ However, there is no current recommendation for assessment of nutritional status in patients with HCC.

A prospective study identified malnutrition as an independent negative prognostic factor in HCC, but interventional studies addressing the effect of nutritional therapy in HCC patients are few. ${ }^{21}$ Huang et al. ${ }^{22}$ reported that malnutrition was associated with an increased risk of postoperative complications and a prolonged length of hospital stay following hepatectomy in patients with $\mathrm{HCC}^{23}$ In patients undergoing curative resection for HCC, perioperative nutritional management has been identified as a key determinant of treatment success. Consequently, there is an urgent need for an objective and simple laboratory test to evaluate malnutrition.

\section{EVALUATION OF NUTRITIONAL INDEXES}

The currently available screening tools for malnutrition include anthropometric measurements, subjective global assessment (SGA), the Nutritional Risk Screening (NRS 2002) score, the mini nutritional assessment (MNA) score, laboratory work-up, and bioelectrical impedance analysis (BIA) measurement for sarcopenia.
The SGA and anthropometric measurements are useful screening tools for nutritional status assessment, but are not suitable for patients with liver cirrhosis. In addition, ascites and edema frequently impede determination of body mass index (BMI), hence BMI is not suitable for identification of malnourished patients with HCC. ${ }^{24-26}$

The NRS 2002 is a tool developed by Kondrup et al. ${ }^{27}$ and the European Society for Clinical Nutrition and Metabolism working group. It includes measurements of current potential undernutrition and disease severity, and correlates them with clinical outcomes of cancer patients in multivariate analyses. ${ }^{28}$ The MNA score is a simple and quick method developed by Vellas et al. ${ }^{29}$ for the assessment of nutritional status in elderly patients. In patients with liver cancer, the MNA can be an effective tool for assessing the nutritional status of patients with liver cancer, but data regarding the impact on patient clinical outcomes have not been published yet. ${ }^{30}$

The BIA electronically measures body composition and serves as an objective, reliable, and non-invasive method for malnutrition assessment in HCC patients. ${ }^{26}$ Anthropometric measurement is not reliable for assessing malnutrition in HCC patients.

The SGA, NRS 2002, and MNA are designed to require investigators to evaluate nutritional issues through individual interviews with patients, requiring time-consuming and skilled personnel. BIA and sarcopenia measurements also require experts to operate the necessary equipment. However, the CONUT score is an objective and simple laboratory test for evaluation of malnutrition.

The PNI is calculated from the serum albumin level and total lymphocyte count, and has conventionally been used to evaluate immune-nutritional status and surgical risk in gastrointestinal surgery. ${ }^{31}$ In addition, the PNI is a significant prognostic factor for evaluating short-term outcomes or overall survival (OS) of HCC patients after hepatectomy. ${ }^{32,33}$ A recent report suggested that the CONUT score predicted survival in colorectal cancer patients more accurately than the PNI. ${ }^{34}$

The CONUT is a newly proposed scoring system, first reported by Ignacio de Ulíbarri et al. ${ }^{35}$ in 2005 as a screening tool for early detection and continuous control of hospital 
undernutrition. The CONUT is calculated from three blood parameters: serum albumin, total peripheral lymphocyte counts, and total cholesterol concentration, which may be readily determined from a venous blood sample. The PNI and CONUT scores both involve measurement of serum albumin and total lymphocyte count. The serum albumin level is an indicator of protein reserves, and is a strong marker of host nutritional status as well as non-nutritional factors including hepatic functional reserve and inflammation. ${ }^{36}$ The total lymphocyte count serves as an indicator of impaired immune defenses due to undernutrition, and is also a reliable marker reflecting immune and nutritional status in patients with HCC. ${ }^{37}$ Unlike the PNI, the CONUT score includes the cholesterol level, which is a parameter of caloric depletion and considered a prognostic predictor of surgical outcome after hepatectomy for HCC. ${ }^{38,39}$ The combination of three variables in the CONUT score, including serum total cholesterol, may more accurately reflect immune and nutritional conditions. Since the CONUT score can predict survival, its application is advisable to provide preoperative nutritional support to high-risk patients.

The CONUT score, summarized in Table 1 , and as described above includes serum albumin, total lymphocyte counts, and total cholesterol levels. ${ }^{27}$ Patients with CONUT scores of 0-1 have a normal nutritional status, those with CONUT scores of 2-4 are at mild risk, those with CONUT scores of 5-8 are at moderate risk, and those with CONUT scores of 9-12 are at severe risk of malnutrition.

\section{CHARACTERISTICS OF THE SELECTED STUDIES}

Most recently published studies have been retrospective, and conducted mainly in Japan and China. Study characteristics are shown in Table 2. The CONUT cut-off criteria included original cut-off methods and study-specific cut-off methods. The various studies determined cut-off values for CONUT scores by dividing patients into 2 groups: 1 ) CONUT $\leq 1$ vs. CONUT $\geq 2$ in one study, ${ }^{40} 2$ ) CONUT $\leq 2$ vs. CONUT $\geq 3$ in one study, ${ }^{41} 3$ ) CONUT $\leq 3$ vs. CONUT $\geq$ 4 in 2 studies, ${ }^{42,43}$ and CONUT $\leq 4$ vs. CONUT $\geq 5$ in one study. ${ }^{44}$ The HCC prevalence in patients with high CONUT scores ranged from 9.1-49.2\%.

\section{POSTOPERATIVE OUTCOMES}

Postoperative outcomes based on the CONUT scores are summarized in Table 3. One study by Li et al. ${ }^{45}$ reported significant differences in postoperative complications according to CONUT scores, and found that postoperative complications after hepatectomy were closely associated with hepatectomyrelated mortality. The study investigated the relationship between CONUT scores and postoperative complications in 1,334 HCC patients who underwent hepatectomy. A CONUT score of 8 was identified as the cut-off value. The patients were divided into two groups: the high-score group where CONUT $\geq 8$, and the low- score group where CONUT $<8$. Based on multivariate analysis, an early postoperative CONUT $\geq 8$ (odds

Table 1. CONUT score for assessment of undernutrition status

\begin{tabular}{lcccc}
\hline & \multicolumn{2}{c}{ Undernutrition status } \\
\cline { 2 - 4 } & Normal & Light & Moderate & Severe \\
\hline Albumin $(\mathrm{g} / \mathrm{dL})$ & $\geq 3.5$ & $3.0-3.49$ & $2.5-2.9$ & $<2.5$ \\
Score & 0 & 2 & 4 & 6 \\
Total lymphocytes $\left(/ \mathrm{mm}^{3}\right)$ & $>1,600$ & $1,200-1,599$ & $800-1,199$ & $<800$ \\
Score & 0 & 1 & 2 & 3 \\
Total cholesterol $(\mathrm{mg} / \mathrm{dL})$ & $>180$ & $140-180$ & $100-139$ & $<100$ \\
Score & 0 & 1 & 2 & 3 \\
Total score & $0-1$ & $2-4$ & $5-8$ & $9-12$ \\
\hline
\end{tabular}

CONUT, controlling nutritional status. 
ratio $[\mathrm{OR}]=2.054,95 \%$ confidence interval $[\mathrm{CI}]=1.371-3.078$, $P<0.001)$, and transfusion $(\mathrm{OR}=3.235,95 \% \mathrm{CI}=2.159-4.847$, $P<0.001)$ were identified as independent risk factors for major postoperative complications. $^{45}$

Wang et al. ${ }^{46}$ reported that a high CONUT score was a predictor of poor survival and postoperative hepatitis B virus reactivation in $\mathrm{HBV}$-related HCC patients with low HBVDNA levels of $<500$ copies $/ \mathrm{mL}$. They insisted that greater care should be taken with low HBV-DNA patients with impaired immune-nutrition status since further antiviral therapy might be necessary.

Lin et al. ${ }^{40}$ conducted a retrospective study on $380 \mathrm{HBV}$ associated HCC patients who underwent hepatectomy between 2006 and 2012. They divided patients into two groups, those with a CONUT score $\geq 2$, and those with a score $<2$. Results showed that the high-score group had a poorer 5 -year survival curve than the low-score group (66.7\% vs.
$82.8 \%$, respectively, $P=0.001$ ). The 5-year recurrence-free survival (RFS) rates were also worse in the high-score group than in the low-score group $(37.2 \%$ vs. $47.6 \%$, respectively, $P=0.016)$. The authors demonstrated that the CONUT score was a better predictor of survival than the PNI score.

Takagi et al. ${ }^{41}$ reviewed medical records data from 295 patients who underwent hepatectomy for HCC between 2007 and 2014. They divided patients into two groups: high CONUT $\geq 3$ score, and low CONUT $\leq 2$ score. The 2 - and 5 -year RFS rates were $45.8 \%$ and $27.9 \%$, in the high-score group, and $57.6 \%$ and $41.4 \%$, in the low-score group, respectively $(P=0.011)$. The 2 - and 5 -year OS rates following hepatectomy were $77.7 \%$ and $61.9 \%$ in the high-score group and $89.3 \%$ and $74.9 \%$ in the low-score group, respectively. Multivariate analysis showed that five variables were significant factors for a poor prognosis: 1) high CONUT score, 2) American Society of Anesthesiologists physical status of 3 or

Table 2. Selected literatures on the effects of CONUT score in patients who underwent surgery for HCC

\begin{tabular}{|c|c|c|c|c|c|c|c|}
\hline Study & Year & Country & Study design & Cases (male, \%) & $\begin{array}{l}\text { Cut-off for } \\
\text { high-CONUT } \\
\text { group }\end{array}$ & $\begin{array}{l}\text { Prevalence } \\
(\%)\end{array}$ & Cut-off method \\
\hline Takagi et al. ${ }^{41}$ & 2017 & Japan & $\begin{array}{l}\text { Retrospective, } \\
\text { single center }\end{array}$ & $295(241,81.7)$ & $\geq 3$ & 40 & NA \\
\hline Harimoto et al. ${ }^{42}$ & 2017 & Japan & $\begin{array}{l}\text { Retrospective, } \\
\text { single center }\end{array}$ & $357(270,75.6)$ & $\geq 4$ & 19.3 & ROC analysis \\
\hline Harimoto et al. ${ }^{43}$ & 2018 & Japan & $\begin{array}{l}\text { Retrospective, } \\
\text { multi-center }\end{array}$ & $2,461(1,785,72.5)$ & $\geq 4$ & 21.9 & ROC analysis \\
\hline Takagi et al. ${ }^{48}$ & 2019 & Japan & $\begin{array}{l}\text { Retrospective, } \\
\text { single center }\end{array}$ & $331(269,81.3)$ & $\geq 5$ & 9.1 & Original cut-off \\
\hline Lin et al. ${ }^{40}$ & 2019 & China & $\begin{array}{l}\text { Retrospective, } \\
\text { single center }\end{array}$ & $380(333,87.6)$ & $\geq 2$ & 49.2 & ROC analysis \\
\hline
\end{tabular}

CONUT, controlling nutritional status; HCC, hepatocellular carcinoma; NA, not available; ROC, receiver operating characteristic.

Table 3. Studies reporting the effects of CONUT score on postoperative outcomes in HCC patients

\begin{tabular}{|c|c|c|c|}
\hline Study & Groups based on CONUT & $\begin{array}{l}\text { Postoperative complications } \\
\text { major (CDC grade } \geq 3)\end{array}$ & Outcomes \\
\hline Takagi et al. ${ }^{41}$ & CONUT $\leq 2$ vs. CONUT $\geq 3$ & $14.1 \%$ vs. $15.2 \%(P=0.79)$ & $5 y r-O S 74.9 \%$ vs. $61.9 \%, 5 y r-R F S 41.4 \%$ vs. $27.9 \%$ \\
\hline Harimoto et al. ${ }^{42}$ & CONUT $\leq 3$ vs. CONUT $\geq 4$ & $14.9 \%$ vs. $20.3 \%(P=0.36)$ & $5 y r-O S 78.0 \%$ vs. $47.6 \%, 5 y r-R F S 38.0 \%$ vs. $8.8 \%$ \\
\hline Harimoto et al..$^{43}$ & CONUT $\leq 3$ vs. CONUT $\geq 4$ & $11.0 \%$ vs. $17.7 \%(P<0.01)$ & Not measured \\
\hline Takagi et al. ${ }^{48}$ & CONUT $\leq 4$ vs. CONUT $\geq 5$ & $13.6 \%$ vs. $23.3 \%(P=0.15)$ & In-hospital mortality $1.3 \%$ vs. $10.0 \%(P=0.002)$ \\
\hline Lin et al. ${ }^{40}$ & CONUT $<2$ vs. CONUT $\geq 2$ & $23.3 \%$ vs. $29.4 \%(P=0.177)$ & $5 y r-O S 82.8 \%$ vs. $66.7 \%, 5 y r-R F S 47.6 \%$ vs. $37.2 \%$ \\
\hline
\end{tabular}

CONUT, controlling nutritional status; CDC, Clavien-Dindo classification; OS, overall survival; RFS, recurrence-free survival. 
4, 3) Child-Pugh score B, 4) presence of multiple tumors, and 5) microvascular invasion. They found a strong correlation between CONUT score and survival, with high-score patients demonstrating significantly lower RFS and OS. ${ }^{41}$

Takagi et al. ${ }^{44}$ also reviewed clinical data from 331 patients who underwent hepatectomy for HCC between 2007 and 2015. Patients were divided into two groups, those with a high CONUT $\geq 5$ score and those with a low CONUT $\leq 4$ score. The high-score group had significantly higher incidences of 30-day mortality $(P<0.001)$, in-hospital mortality ( $P=0.002)$, ascites $(P=0.006)$, post-hepatectomy liver failure $(P=0.02)$, sepsis $(P=0.01)$, and enteritis $(P<0.001)$ compared to the low-score group. They also found a significant relationship between the CONUT score and various clinical parameters such as the Child-Turcotte-Pugh score, platelet count, prothrombin time, technetium-99m diethylenetriamine-penta-acetic acid-galactosyl human serum albumin value, and degree of fibrosis. Notably, PNI was not an independent predictor in this study. Therefore, the CONUT score may be a more accurate prognostic factor than the PNI since the CONUT score also includes total cholesterol level. Moreover, a high CONUT score was more helpful than a PNI score for evaluating patient nutritional status and mortality risk after liver surgery. Consequently, the authors concluded that compared with other scoring systems (such as the PNI), the CONUT score was more accurate and objective, and performed better as a prognostic marker. ${ }^{44}$

Harimoto et al. ${ }^{42}$ retrospectively reviewed the survival rate of 357 patients who underwent hepatectomy for HCC between 2004 and 2015. The optimal cut-off value for the preoperative CONUT score calculated by ROC curve analysis was 3. Patients were assigned to two groups, those with a CONUT score $>3$ (high-score group) and patients with a score $\leq 3$ (low-score group). The 5-year OS rates of patients with low and high CONUT scores were $78.0 \%$ and $47.6 \%$, respectively, and the 5 -year RFS rates were $38.0 \%$ and $8.8 \%$, respectively. Multivariate analysis identified six prognostic factors for reduced OS: 1) older age, 2) liver damage B, 3) high CONUT score, 4) poor tumor differentiation, 5) intrahepatic metastasis, and 6) blood transfusion. Additionally, five prognostic factors were identified for reduced RFS in- cluding: 1) older age, 2) higher indocyanin green retention rate at 15 minutes, 3) larger tumor size, 4) intrahepatic metastasis, and 5) blood transfusion. The authors concluded that CONUT scores were independently associated with OS, but not RFS, in HCC patients undergoing hepatectomy. ${ }^{42}$

Harimoto et al. ${ }^{43}$ also performed a multi-institutional retrospective analysis of 2,461 patients who underwent curative resection for HCC. They applied an optimal cut-off value for the preoperative CONUT score of 4 . Of the 2,461 patients, 54 (21.9\%) had preoperative CONUT scores $\geq 4$ (the highscore group). After propensity score matching, the prognosis for OS and RFS in patients with high CONUT scores was significantly worse than that for patients with low CONUT scores. The selected cut-off in the study indicated severe preoperative immune and nutritional status in many patients with liver cirrhosis, and therefore the authors presumed that sarcopenia was related to nutritional status or host immune status, and might lead to a high CONUT score.

Liu et al. ${ }^{47}$ reported that a systemic inflammation-based score can predict survival in intermediate- to advanced-HCC patients receiving transcatheter arterial chemoembolization (TACE). Hence, the CONUT score could help predict the survival rate of HCC patients undergoing TACE, but further study is needed for confirmation.

Previous meta-analyses have reported that long-term outcomes for gastrointestinal and solid tumor patients with high CONUT scores are poor. ${ }^{48,49}$ However, these studies had limitations. First, the number of included papers was relatively small, and included few HCC meta-analyses mostly conducted in Japan and China. Accordingly, the results might not be generalizable to other populations. Second, most of the papers were retrospective studies and applied different CONUT score cut-off values. In future, standardization is essential, and more studies are needed, especially from Western countries, to confirm the effects of CONUT scores on HCC outcomes.

Unlike other cancers, HCC patients typically also have liver cirrhosis. However, most of the patients undergoing surgery are patients with Child-Pugh A scores, so their albumin levels are often normal. Thus, we speculate that in HCC, the CONUT score might be a more comprehensive and superior 
predictor for nutritional risk than the PNI score.

\section{CONCLUSIONS}

Malnutrition was associated with unfavorable outcomes in patients who underwent hepatectomy for HCC. Evaluation of nutritional status may provide additional prognostic information. This study suggests that the CONUT score is a valuable preoperative predictor of survival in patients with HCC. Alimentary intervention in patients with high CONUT scores can improve nutritional status and long-term survival. However, future multi-institutional prospective studies are required for confirmation.

\section{Conflicts of Interest}

The author declares no conflicts of interest pertaining to this work.

\section{REFERENCES}

1. Bray F, Ren JS, Masuyer E, Ferlay J. Global estimates of cancer prevalence for 27 sites in the adult population in 2008. Int J Cancer 2013;132:1133-1145.

2. Alberino F, Gatta A, Amodio P, Merkel C, Di Pascoli L, Boffo G, et al. Nutrition and survival in patients with liver cirrhosis. Nutrition 2001;17:445-450.

3. Takenaka K, Kawahara N, Yamamoto K, Kajiyama K, Maeda T, Itasaka $\mathrm{H}$, et al. Results of 280 liver resections for hepatocellular carcinoma. Arch Surg 1996;131:71-76.

4. Taketomi A, Kitagawa D, Itoh S, Harimoto N, Yamashita Y, Gion T, et al. Trends in morbidity and mortality after hepatic resection for hepatocellular carcinoma: an institute's experience with 625 patients. J Am Coll Surg 2007;204:580-587.

5. Forner A, Llovet JM, Bruix J. Hepatocellular carcinoma. Lancet 2012;379:1245-1255.

6. Huitzil-Melendez FD, Capanu M, O'Reilly EM, Duffy A, Gansukh B, Saltz LL, et al. Advanced hepatocellular carcinoma: which staging systems best predict prognosis? J Clin Oncol 2010;28:2889-2895.

7. The Cancer of the Liver Italian Program (Clip) Investigators. A new prognostic system for hepatocellular carcinoma: a retrospective study of 435 patients. Hepatology 1998;28:751-755.

8. Llovet JM, Brú C, Bruix J. Prognosis of hepatocellular carcinoma: the BCLC staging classification. Semin Liver Dis 1999;19:329-338.

9. Man Z, Pang Q, Zhou L, Wang Y, Hu X, Yang S, et al. Prognostic significance of preoperative prognostic nutritional index in hepato- cellular carcinoma: a meta-analysis. HPB (Oxford) 2018;20:888-895.

10. Chan AW, Chan SL, Wong GL, Wong VW, Chong CC, Lai PB, et al. Prognostic nutritional index (PNI) predicts tumor recurrence of very early/early stage hepatocellular carcinoma after surgical resection. Ann Surg Oncol 2015;22:4138-4148.

11. Okamura Y, Ashida R, Ito T, Sugiura T, Mori K, Uesaka K. Preoperative neutrophil to lymphocyte ratio and prognostic nutritional index predict overall survival after hepatectomy for hepatocellular carcinoma. World J Surg 2015;39:1501-1509.

12. Baracos VE. Cancer-associated malnutrition. Eur J Clin Nutr 2018;72:1255-1259.

13. Alwarawrah Y, Kiernan K, Maclver NJ. Changes in nutritional tatus impact immune cell metabolism and function. Front Immunol 2018;9:1055

14. Lien YC, Hsieh CC, Wu YC, Hsu HS, Hsu WH, Wang LS, et al. Preoperative serum albumin level is a prognostic indicator for adenocarcinoma of the gastric cardia. J Gastointest Surg 2004;8:1041-1048.

15. Ray-Coquard I, Cropet C, Van Glabbeke M, Sebban C, Le Cesne A, Judson I, et al. Lymphopenia as a prognostic factor for overall in advanced carcinomas, sarcomas, and lymphomas. Cancer Res 2009;69:5383-5391.

16. Schwegler I, von Holzen A, Gutzwiller JP, Schlumpf R, Mühlebach $S$, Stanga Z. Nutritional risk is a clinical predictor of postoperative mortality and morbidity in surgery for colorectal cancer. Br J Surg 2010;97:92-97.

17. Bozzetti F, Mariani L, Lo Vullo S, Amerio ML, Biffi R, Caccialanza G, et al. The nutritional risk in oncology: a study of 1,453 cancer outpatients. Support Care Cancer 2012;20:1919-1928.

18. Jensen $G L$, Bistrian $B$, Roubenoff $R$, Heimburger DC. Malnutrition syndromes: a conundrum vs continuum. J Parenter Enteral Nutr 2009;33:710-716.

19. Montano-Loza AJ, Duarte-Rojo A, Meza-Junco J, Baracos VE, Sawyer MB, Pang JXQ, et al. Inclusion of Sarcopenia within MELD (MELD-sarcopenia) and the prediction of mortality in patients with cirrhosis. Clin Transl Gastroenterol 2015;6:e102.

20. Calmet $F$, Martin P, Pearlman M. Nutrition in patients with cirrhosis. Gastroenterol Hepatol (N Y) 2019;15:248-254.

21. Schütte $K$, Tippelt $B$, Schulz $C$, Röhl F, Feneberg A, Seidensticker $R$, et al. Malnutrition is a prognostic factor in patients with hepatocellular carcinoma (HCC). Clin Nutr 2015;34:1122-1127.

22. Huang TH, Hsieh CC, Kuo LM, Chang C, Chen C, Chi C, et al. Malnutrition associated with an increased risk of postoperative complications following hepatectomy in patients with hepatocellular carcinoma. HPB (Oxford) 2019;21:1150-1155.

23. Ciuni $R$, Biondi $A$, Grosso G, Nunnari G, Panascia E, Randisi L, et al. Nutritional aspects in patient undergoing liver resection. Updates Surg 2011;63:249-252.

24. Kawaguchi T, Taniguchi E, Itou M, Ibi R, Okada T, Mutou M, et al. Body cell mass is a useful parameter for assessing malnutrition and severity of disease in non-ascitic cirrhotic patients with hepatocel- 
lular carcinoma or esophageal varices. Int J Mol Med 2008;22:589594.

25. Alvares-da-Silva MR, Reverbel da Silveira T. Comparison between handgrip strength, subjective global assessment, and prognostic nutritional index in assessing malnutrition and predicting clinical outcome in cirrhotic outpatients. Nutrition 2005;21:113-117.

26. Schütte K, Tippelt B, Schulz C, Röhl FW, Feneberg A, Seidensticker R, et al. Malnutrition is a prognostic factor in patients with hepatocellular carcinoma (HCC). Clin Nutr 2015;34:1122-1127.

27. Kondrup J, Rasmussen HH, Hamberg O, Stanga Z. Nutritional risk screening (NRS 2002): a new method based on an analysis of controlled clinical trials. Clin Nutr 2003;22:321-336.

28. Sorensen J, Kondrup J, Prokopowicz J, Schiesser M, Krahenbühl $L$, Meier $R$, et al. Euro00PS: an international, multicentre study to implement nutritional risk screening and evaluate clinical outcome. Clin Nutr 2008;27:340-349.

29. Vellas B, Guigoz Y, Garry PJ, Nourhashemi F, Bennahum D, Lauque S, et al. The mini nutritional assessment (MNA) and its use in grading the nutritional state of elderly patients. Nutrtion 1999;15:116-122.

30. Tsai AC, Hsu W, Chan S, Chang T. Usefulness of the mini nutritional assessment in predicting the nutritional status of patients with liver cancer in Taiwan. Nutr Cancer 2011;63:334-341.

31. Jiang N, Deng JY, Ding XW, Ke B, Liu Ning, Zhang RP, et al. Prognostic nutritional index predicts postoperative complications and long-term outcomes of gastric cancer. World I Gastroenterol 2014;20:10537-10544.

32. Pinato DJ, North BV, Sharma R. A novel, externally validated inflammation based prognostic algorithm in hepatocellular carcinoma: the prognostic nutritional index (PNI). Br J Canc 2012;106:1439-1445.

33. Ke M, Xu T, Li N, Ren Y, Shi A, Lv Y, et al. Prognostic nutritional index predicts short-term outcomes after liver resection for hepatocelIular carcinoma within the Milan criteria. Oncotarget 2016;7:8161181620.

34. Iseki Y, Shibutani M, Maeda K, Nagahara H, Ohtani H, Sugano K, et al. Impact of the preoperative controlling nutritional status (CONUT) score on the survival after curative surgery for colorectal cancer. PLoS One 2015;10:e0132488.

35. Ignacio de Ulíbarri J, González-Madroño A, de Villar NG, González P, González B, Mancha A, et al. CONUT: a tool for controlling nutritional status. First validation in a hospital population. Nutr Hosp 2005;20:38-45.

36. Oñate-Ocaña LF, Aiello-Crocifoglio V, Gallardo-Rincón D, HerreraGoepfert R, Brom-Valladares R, Carrillo JF, et al. Serum albumin as a significant prognostic factor for patients with gastric carcinoma. Ann Surg Oncol 2007;14:381-389.

37. Lu SD, Wang YY, Peng NF, Peng YC, Zhong JH, Qin HG, et al. Preoperative ratio of neutrophils to lymphocytes predicts postresection survival in selected patients with early or intermediate stage hepatocellular carcinoma. Medicine (Baltimore) 2016;95:e2722.

38. Jiang SS, Weng DS, Jiang L, Zhang YJ, Pan K, Pan QZ, et al. The clini- cal significance of preoperative serum cholesterol and high-density lipoprotein-cholesterol levels in hepatocellular carcinoma. J Cancer 2016;7:626-632.

39. Lee YL, Li WC, Tsai TH, Chiang HY, Ting CT. Body mass index and cholesterol level predict surgical outcome in patients with hepatocellular carcinoma in Taiwan-a cohort study. Oncotarget 2016;7:2294822959.

40. Lin ZX, Ruan DY, Jia CC, Wang TT, Cheng JT, Huang HQ, et al. Controlling nutritional status (CONUT) score-based nomogram to predict overall survival of patients with HBV-associated hepatocellular carcinoma after curative hepatectomy. Clin Transl Oncol 2020;22:370380.

41. Takagi K, Yagi T, Umeda Y, Shinoura S, Yoshida R, Nobuoka D, et al. Preoperative controlling nutritional status (CONUT) score for assessment of prognosis following hepatectomy for hepatocellular carcinoma. World J Surg 2017;41:2353-2360.

42. Harimoto N, Yoshizumi T, Sakata K, Nagatsu A, Motomura T, Itoh S, et al. Prognostic significance of preoperative controlling nutritional status (CONUT) score in patients undergoing hepatic resection for hepatocellular carcinoma. World J Surg 2017;41:2805-2812.

43. Harimoto N, Yoshizumi T, Inokuchi S, Itoh S, Adachi E, Ikeda Y, et al. Prognostic significance of preoperative controlling nutritional status (CONUT) score in patients undergoing hepatic resection for hepatocellular carcinoma: a multi-institutional study. Ann Surg Oncol 2018:25:3316-3323.

44. Takagi K, Umeda Y, Yoshida R, Nobuoka D, Kuise T, Fushimi T, et al. Preoperative controlling nutritional status score predicts mortality after hepatectomy for hepatocellular carcinoma. Dig Surg 2019;36:226-232.

45. Li L, Liu C, Yang J, Wu H, Wen T, Wang W, et al. Early postoperative controlling nutritional status (CONUT) score is associated with complication III-V after hepatectomy in hepatocellular carcinoma: a retrospective cohort study of 1,334 patients. Sci Rep 2018;8:13406.

46. Wang XB ,Chen J, Xiang BD, Wu FX, Li LQ. High CONUT score predicts poor survival and postoperative HBV reactivation in HBVrelated hepatocellular carcinoma patients with low HBV-DNA levels. Eur J Surg Oncol 2019;45:782-787.

47. Liu C, Li L, Lu WS, Du H, Yan LN, Wen TF, et al. A novel combined systemic inflammation-based score can predict survival of intermediate-to-advanced hepatocellular carcinoma patients undergoing transarterial chemoembolization. BMC Cancer 2018;18:216.

48. Takagi K, Domagala P, Polak WG, Buettner S, ljzermans JNM. The controlling nutritional status score and postoperative complication risk in gastrointestinal and hepatopancreatobiliary surgical oncology: a systematic review and meta-analysis. Ann Nutr Metab 2019;74:303-312.

49. Zhang $Y$, Zhang $X$. Controlling nutritional status score, a promising prognostic marker in patients with gastrointestinal cancers after surgery: a systematic review and meta-analysis. Int J Surg 2018;55:3945. 